\title{
Maternal Physical Activity and Neonatal Cord Blood Lipid Levels: Findings From a Prospective Pregnancy Cohort
}

\author{
Paul J. Collings, Diane Farrar, Joanna Gibson, Jane West, Sally E. Barber, and John Wright
}

\begin{abstract}
Background: Physical activity performed while pregnant is beneficially associated with maternal cardiovascular health. It is unknown if benefits extend to neonatal cardiovascular health. This study investigated associations of maternal physical activity with neonatal cord blood lipid and lipoprotein concentrations. Methods: Cord blood lipids were measured at birth in a pseudorandomly selected subgroup of Born in Bradford birth cohort participants $(\mathrm{N}=1634)$. Pregnant women were grouped into 4 activity categories (inactive/somewhat active/moderately active/active) based on their self-reported physical activity at 26- to 28-weeks gestation. Regression was used to calculate adjusted mean differences in neonatal cord blood lipid concentrations among the 4 groups of physical activity. Results: Maternal physical activity was associated with higher neonatal cord blood high-density lipoprotein cholesterol. Cord blood high-density lipoprotein cholesterol was higher in neonates of women who were somewhat and moderately active compared with neonates of women who were inactive. There were no associations of pregnancy physical activity with triglycerides, low-density lipoprotein, very low-density lipoprotein cholesterol, or adiponectin levels. Conclusions: Maternal physical activity is favorably associated with neonatal cord blood high-density lipoprotein cholesterol levels. This novel beneficial finding highlights the potential for physical activity in pregnancy to aid the early prevention of cardiovascular disease.
\end{abstract}

Keywords: exercise, fetal blood, metabolic diseases, adiponectin, fetal development

Physical activity confers myriad benefits to the uncomplicated pregnancy, ${ }^{1}$ benefits that can have long-term health impacts for the developing fetus. ${ }^{2,3}$ Our understanding that intrauterine environments can program susceptibility to future disease has developed over the last 2 decades. ${ }^{4}$ This work has highlighted that in-utero exposures can influence the risk of cardiovascular disease (CVD), ${ }^{5}$ the leading cause of global morbidity ${ }^{6}$ and mortality. ${ }^{7}$ We recently reported that maternal physical activity is favorably associated with lower levels of maternal pregnancy triglycerides and a higher concentration of high-density lipoprotein cholesterol (HDL-c), ${ }^{8}$ established precursors of CVD. ${ }^{9}$ It is unknown if maternal physical activity confers similar benefits, that is a more favorable blood lipid profile, to the developing fetus. This is important to investigate, as cord blood lipid profiles track over time to early childhood ${ }^{10}$ (when they can begin to initiate and progress preclinical signs of $\mathrm{CVD}^{11}$ ), and subsequently to adulthood ${ }^{12}$ when the clinical manifestations of CVD arise. ${ }^{13}$ This study investigated associations of maternal physical activity with neonatal cord blood lipid levels.

\section{Methods}

Born in Bradford (BiB) is a prospective birth cohort study of 12,453 women who were recruited at 26- to 28 -weeks gestation and who delivered 13,818 live births between 2007 and 2010. Full study details are provided elsewhere. ${ }^{14}$ In a pseudorandomly selected subgroup of BiB participants, cord blood lipid samples were collected. ${ }^{15}$ For the purposes of this complete case investigation, the subgroup was restricted to singleton pregnancies and

Collings, Farrar, Gibson, West, Barber, and Wright are with the Bradford Institute for Health Research, Bradford Teaching Hospitals NHS Foundation Trust, Bradford, United Kingdom. Collings is also with the Department of Health Sciences, University of York, York, United Kingdom. Collings (paul.collings@bthft.nhs. $\mathrm{uk}$ ) is corresponding author. women who were free from preexisting hypertension and diabetes before pregnancy. The final sample comprised 1634 mother-neonate pairs. Characteristics of the included subgroup were similar to those of all other BiB participants (Supplementary Table 1 [available online]), who were broadly representative of the obstetric population in Bradford at the time of recruitment. ${ }^{14}$ The BiB study was approved by the Bradford Research Ethics Committee (ref 07/ H1302/112), and all mothers provided written informed consent.

Maternal physical activity was assessed at recruitment using the General Practice Physical Activity Questionnaire, which has been validated against accelerometry ${ }^{16}$ and exhibits face validity in the $\mathrm{BiB}$ cohort. ${ }^{8}$ Mothers were assigned to 1 of 4 activity levels (inactive/somewhat active/moderately active/active) based on their self-reported occupational physical activity level, physical exercise, and walking in the last week. The active category is consistent with meeting the recommended minimum of $150 \mathrm{~min} / \mathrm{wk}$ of moderate-intensity physical activity. ${ }^{17}$

Cord blood samples were obtained at delivery by the attending midwife. Samples were refrigerated at $4^{\circ} \mathrm{C}$ in EDTA tubes until collected by laboratory staff within 12 hours. Samples were then spun, frozen, and stored at $-80^{\circ} \mathrm{C} .{ }^{15}$ Following transfer to the Biochemistry Department of Glasgow Royal Infirmary, enzymatic reagents were used to determine serum concentrations of total cholesterol, triglycerides, HDL-c, low-density lipoprotein cholesterol (LDL-c), and very low-density lipoprotein cholesterol (Cobas C311 autoanalyzer; Roche Diagnostics, Basel, Switzerland). Cord blood adiponectin was measured using commercially available ELISA kits (Quantikine human adiponectin immunoassay; R\&D Systems, Minneapolis, MN).

Women consented to the abstraction and use of data from their obstetric medical records and at recruitment completed an intervieweradministered questionnaire. The questionnaire collated information regarding ethnicity, social and economic circumstances, smoking, alcohol, caffeine intake, and sleep. Interviews were conducted in a variety of languages. Maternal weight at $\sim 12$-weeks gestation was combined with height to derive early pregnancy body mass index 
(in kilograms per meter squared). Gestational age at birth was calculated as the number of weeks elapsed between conception (based on ultrasound examination at $\sim 12$-weeks) and delivery. Full details of covariables are available elsewhere. ${ }^{8}$

For description, participant characteristics were summarized by maternal pregnancy physical activity level, and Pearson correlation coefficients were calculated between maternal blood lipid levels (measured at recruitment ${ }^{8}$ ) and cord blood lipid concentrations. For the main analysis, linear regression models were used to calculate differences in cord blood lipid concentrations among the 4 groups of maternal physical activity (reference group: inactive); $p$ values from trend tests across physical activity categories are also presented. Models were initially adjusted for maternal age, ethnicity, early pregnancy body mass index, socioeconomic status, parity, season of physical activity assessment, and neonate sex. Adjustments for maternal smoking in pregnancy, delivery mode, birth weight, and gestational age were subsequently added as they changed $\beta$ coefficients between exposures and outcomes by $\geq 10 \% .{ }^{18}$ All cord blood lipid distributions were skewed and were natural log transformed prior to analyses; the data have been back-transformed by exponentiation. Results are presented as marginal means with 95\% confidence intervals. Analyses were performed in Stata/SE software (version 15.0; Statacorp, College Station, TX).

\section{Results}

Descriptive statistics for the cord blood lipid subgroup are presented in Table 1. More than half of women (60.4\%) were inactive, one-fifth were somewhat active (18.2\%), and fewer were classified as moderately active (12.0\%) and active (9.4\%), respectively. Inactive women were more frequently of Pakistani origin, multiparous, and were moderately or most deprived. Correlations between maternal and cord blood lipids were calculable for 1510 mother-neonate pairs and were consistently weak (total cholesterol, 0.08; HDL-c, 0.16; LDL-c, 0.06; triglycerides, 0.08).

Table 2 shows neonatal cord blood lipid and adiponectin levels stratified by maternal pregnancy physical activity. There was no strong evidence for effect modification by ethnic group $(P \geq .11)$, so the results are presented for the whole sample combined and adjusted for ethnicity. There was a significant trend across categories to indicate that higher physical activity was associated with higher cord blood total cholesterol; levels were significantly higher in neonates of women who were moderately active in pregnancy compared with neonates of women who were inactive. Analysis of individual components revealed that there was only a positive association of maternal physical activity with cord blood HDL-c; values were significantly higher in neonates of women who were somewhat active and moderately active, respectively, compared with neonates of women who were inactive. For neonates of active women, the confidence interval was consistent with higher cord blood HDL-c in comparison with neonates of inactive women, but the difference was not statistically significant, likely due to type II error caused by fewer observations. There was no evidence for associations of pregnancy physical activity with neonatal cord blood trigylycerides, LDL-c, very low-density lipoprotein cholesterol, or adiponectin concentrations.

\section{Discussion}

This study found that maternal pregnancy physical activity was favorably associated with a higher concentration of neonatal cord blood HDL-c. The association was modest in scale, but may be underestimated due to errors in self-reported physical activity. It is also important to consider that any positive influence on cord blood HDL-c may be meaningful because cord blood lipid levels track over time $\mathrm{1}^{10,12}$ and an adverse lipid profile in childhood can initiate preclinical signs of CVD. ${ }^{11}$ Furthermore, even in the absence of any other blood lipid abnormality, low HDL-c has been shown to elevate CVD risk in adulthood. ${ }^{19}$

We are aware of only 2 other studies that have investigated this topic. In a Canadian study of 442 mother-neonate pairs, there was no evidence for an association between self-reported physical activity with a latent parameter that represented cord blood HDL-c, apolipoprotein A1, and LDL-c. ${ }^{20}$ Similarly, no associations with traditional blood lipids were found in 51 women from Austria who underwent an assessment of physical activity by accelerometry at 10- to 14-weeks gestation. However, in that study, unadjusted analyses did show that cord blood exhibited a more favorable composition of HDL-c (lower oxidized HDL-c and higher apolipoprotein A1) in neonates of women who met the recommended level of physical activity compared with those who did not. ${ }^{21}$ The current study is the first to provide evidence of an independent association between pregnancy physical activity with higher cord blood HDL-c. Importantly, compared with neonates of women who were inactive, cord blood HDL-c levels were higher even in neonates of women who were merely somewhat active in pregnancy. Hence, even small volumes of physical activity well below the recommended minimum $(150 \mathrm{~min} / \mathrm{wk}$ of moderateintensity physical activity) were beneficial. This supports current UK guidelines that "every activity counts" and that inactive pregnant women should gradually accumulate physical activity throughout the week. ${ }^{17}$

Safety concerns are often cited by pregnant women as a barrier to physical activity. ${ }^{22}$ This may partly explain why fewer than $10 \%$ of our study participants were active. Reassuringly, the results of this study, coupled with previous observations made in the same cohort, ${ }^{8}$ add to growing evidence that pregnancy physical activity is not only safe but is beneficial for the short- and long-term health prospects of both mother and child. Women should be encouraged to be physically active while pregnant and reassured that evidence suggests that it is safe. A recent systematic review concluded that multicomponent interventions can successfully increase pregnancy physical activity levels, with the most promising strategies including group exercise classes, information provision about recommended levels of physical activity and suitable exercises, and guidance about how to overcome perceived barriers including the management of various pregnancy-related symptoms. ${ }^{23}$ This information should also be disseminated to health care professionals who are well placed to advocate physical activity but who may lack the confidence, knowledge, and resources to deliver appropriate and safe advice. ${ }^{24}$ The results of this study further highlight that Pakistani-origin women and women from deprived backgrounds are the most inactive in pregnancy, and thus, they may be considered priority recipients of efforts to increase physical activity. Targeting these women (who are also more susceptible to pregnancy complications ${ }^{25}$ ) could help to reduce health inequalities by reducing the risk of adverse pregnancy outcomes and their long-term sequelae in high-risk groups.

In line with observations made at birth, ${ }^{26}$ we found that maternal blood lipids were weakly correlated with neonatal cord blood lipids. This is reassuring, as it highlights that cord blood lipids represent neonate cardiovascular status (likely as a consequence of fetal and placental influences on cord blood biochemistry) as opposed to merely reflecting maternal lipids. It is a limitation 


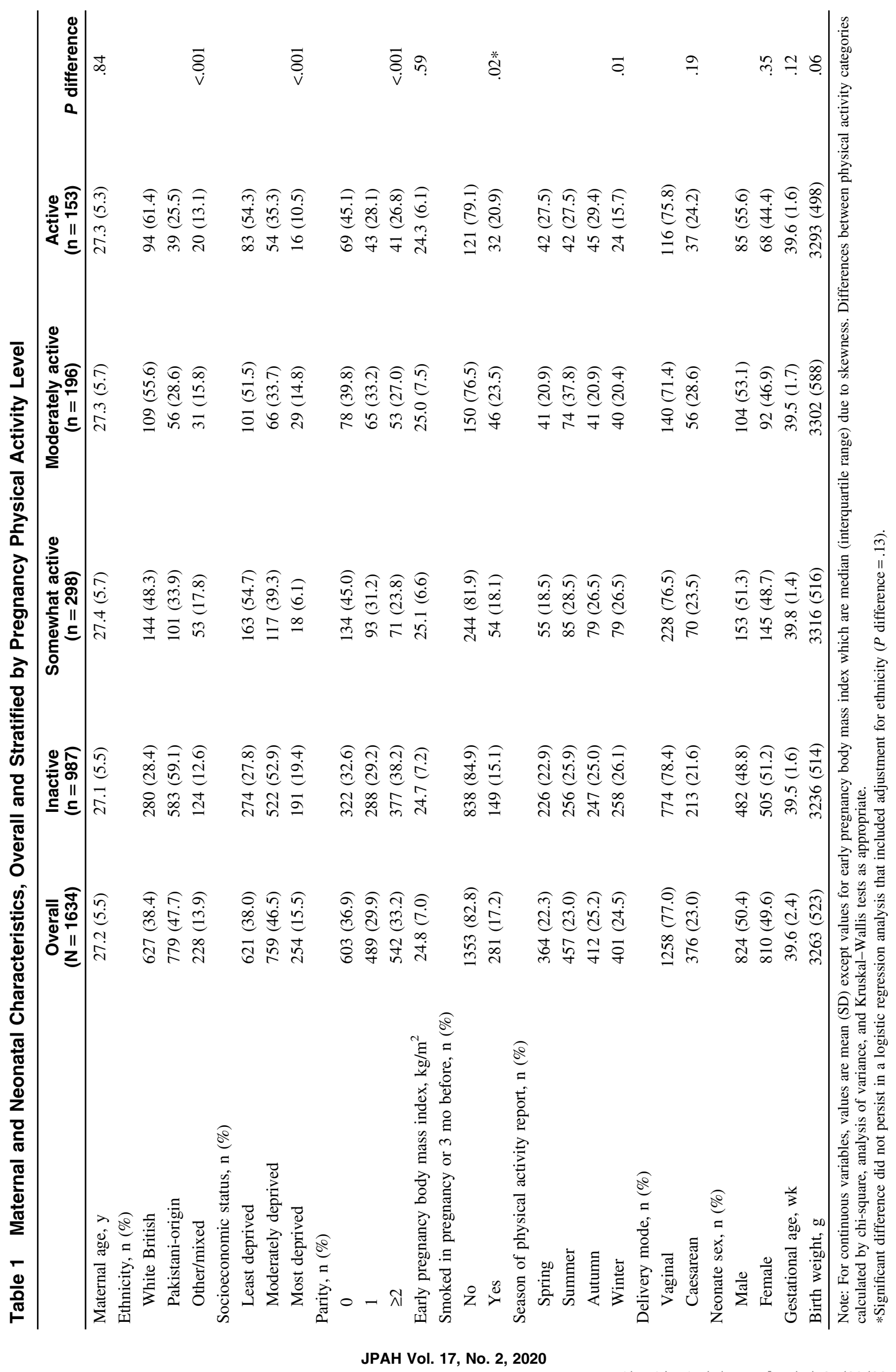




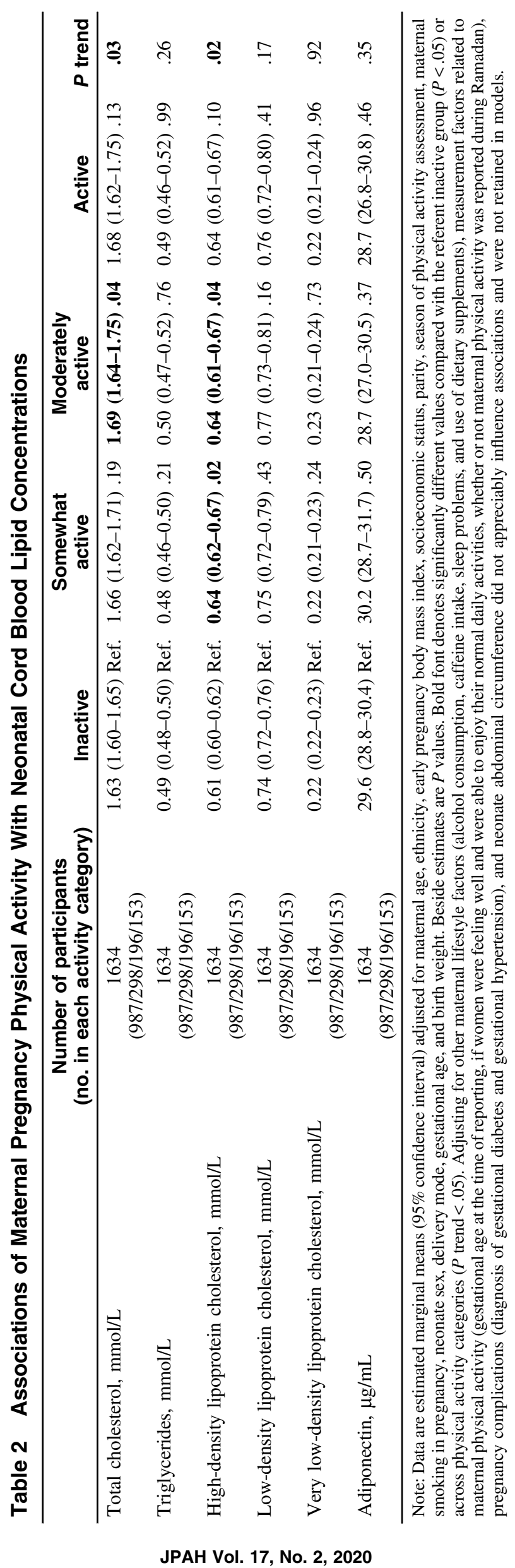


of this study, nevertheless, that information for several covariables was self-reported and that the analyses were not adjusted for maternal dietary factors. Future studies should investigate longer term influences of maternal pregnancy physical activity on offspring cardiovascular health.

\section{Conclusion}

Maternal physical activity is beneficially associated with neonatal cord blood HDL-c levels. This novel finding highlights the potential for promoting physical activity in pregnancy to aid the early prevention of CVD.

\section{Acknowledgments}

Born in Bradford (BiB) is only possible because of the enthusiasm and commitment of the children and parents in $\mathrm{BiB}$. We are grateful to all the participants, health professionals, and researchers who have made $\mathrm{BiB}$ happen. The BiB study receives core infrastructure funding from the Wellcome Trust (WT101597MA), a joint grant from the UK Medical Research Council (MRC) and UK Economic and Social Science Research Council (ESRC) (MR/N024397/1); the British Heart Foundation (BHF) (CS/16/4/32482); and the National Institute for Health Research (NIHR) under its Collaboration for Applied Health Research and Care (CLAHRC) for Yorkshire and Humber, and Clinical Research Network (CRN). Authors are part of the Healthy Children, Healthy Families Theme of the NIHR CLAHRC for Yorkshire and Humber. J. West was supported by a MRC Population Health Scientist Postdoctoral Award (MR/K021656/1) and P.J.C. is funded by a BHF Immediate Postdoctoral Basic Science Research Fellowship (FS/17/37/32937). The views expressed in this paper are those of the authors and not necessarily those of the MRC, ESRC, BHF, NIHR, and UK Department of Health or National Health Services or of any other funder acknowledged here. The authors declare that they have no conflict of interest.

\section{References}

1. Dipietro L, Evenson KR, Bloodgood B, et al; 2018 Physical Activity Guidelines Advisory Committee. Benefits of physical activity during pregnancy and postpartum. Med Sci Sports Exerc. 2019;51(6):12921302. doi:10.1249/MSS.0000000000001941

2. Damm P, Houshmand-Oeregaard A, Kelstrup L, Lauenborg J, Mathiesen ER, Clausen TD. Gestational diabetes mellitus and longterm consequences for mother and offspring: a view from Denmark. Diabetologia. 2016;59(7):1396-1399. PubMed ID: 27174368 doi:10.1007/s00125-016-3985-5

3. Catalano PM, Shankar K. Obesity and pregnancy: mechanisms of short term and long term adverse consequences for mother and child. BMJ. 2017;356:j1. PubMed ID: 28179267 doi:10.1136/bmj.j1

4. Barker DJP. The origins of the developmental origins theory. J Intern Med. 2007;261(5):412-417. PubMed ID: 17444880 doi:10.1111/j. 1365-2796.2007.01809.x

5. Yeung EH, Robledo C, Boghossian N, Zhang C, Mendola P. Developmental origins of cardiovascular disease. Curr Epidemiol Rep. 2014;1(1):9-16. PubMed ID: 25364653 doi:10.1007/s40471-0140006-4

6. World Health Organization. Global Health Estimates 2016: Disease Burden by Cause, Age, Sex, by Country and by Region, 2000-2016. Geneva, Switzerland: World Health Organization; 2018.

7. World Health Organization. Global Health Estimates 2016: Deaths by Cause, Age, Sex, by Country and by Region, 2000-2016. Geneva, Switzerland: World Health Organization; 2018.
8. Collings PJ, Farrar D, Gibson J, West J, Barber SE, Wright J. Associations of pregnancy physical activity with maternal cardiometabolic health, neonatal delivery outcomes and body composition in a biethnic cohort of 7305 mother-child pairs: the Born in Bradford study [published online ahead of print September 26, 2019]. Sports Med. doi:10.1007/s40279-019-01193-8

9. Yusuf PS, Hawken S, Ônpuu S, et al. Effect of potentially modifiable risk factors associated with myocardial infarction in 52 countries (the INTERHEART study): case-control study. Lancet. 2004;364(9438): 937-952. PubMed ID: 15364185 doi:10.1016/S0140-6736(04) 17018-9

10. Bastida S, Sánchez-Muniz FJ, Cuena R, Perea S, Aragonés A. High density lipoprotein-cholesterol changes in children with high cholesterol levels at birth. Eur J Pediatr. 2002;161(2):94-98. PubMed ID: 11954759 doi:10.1007/s00431-001-0863-y

11. Pires A, Sena C, Seiça R. Dyslipidemia and cardiovascular changes in children. Curr Opin Cardiol. 2016;31(1):95-100. PubMed ID: 26599060 doi:10.1097/HCO.0000000000000249

12. Juhola J, Magnussen CG, Viikari JSA, et al. Tracking of serum lipid levels, blood pressure, and body mass index from childhood to adulthood: the cardiovascular risk in young Finns study. $J$ Pediatr. 2011;159(4):584-590. PubMed ID: 21514597 doi:10.1016/j.jpeds. 2011.03.021

13. Orozco-Beltran D, Gil-Guillen VF, Redon J, et al. Lipid profile, cardiovascular disease and mortality in a Mediterranean high-risk population: the ESCARVAL-RISK study. PLoS One. 2017;12(10): e0186196. doi:10.1371/journal.pone.0186196

14. Wright J, Small N, Raynor P, et al. Cohort profile: the born in bradford multi-ethnic family cohort study. Int J Epidemiol. 2013; 42(4):978-991. PubMed ID: 23064411 doi:10.1093/ije/dys112

15. Lawlor DA, West J, Fairley L, et al. Pregnancy glycaemia and cordblood levels of insulin and leptin in Pakistani and white British mother-offspring pairs: findings from a prospective pregnancy cohort. Diabetologia. 2014;57(12):2492-2500. PubMed ID: 25273345 doi:10.1007/s00125-014-3386-6

16. National Health Service. The General Practice Physical Activity Questionnaire (GPPAQ) — A screening tool to assess adult physical activity levels, within primary care. https://www.gov.uk/government/ uploads/system/uploads/attachment_data/file/192453/GPPAQ__guidance.pdf. Published 2009.Accessed December 12, 2017.

17. Department of Health and Social Care. UK Chief Medical Officers' physical activity guidelines. 2019. https://www.gov.uk/government/ publications/physical-activity-guidelines-uk-chief-medical-officersreport

18. Maldonado G, Greenland S. Simulation study of confounderselection strategies. Am J Epidemiol. 1993;138(11):923-936. PubMed ID: 8256780 doi:10.1093/oxfordjournals.aje.a116813

19. Huxley RR, Barzi F, Lam TH, et al. Isolated low levels of highdensity lipoprotein cholesterol are associated with an increased risk of coronary heart disease: an individual participant data meta-analysis of 23 studies in the asia-pacific region. Circulation. 2011;124(19): 2056-2064. PubMed ID: 21986289 doi:10.1161/CIRCULATIONAHA. 111.028373

20. Morrison KM, Anand SS, Yusuf S, et al. Maternal and pregnancy related predictors of cardiometabolic traits in newborns. PLoS One. 2013;8(2):e55815. PubMed ID: 23418462 doi:10.1371/journal.pone. 0055815

21. Tatrai K. The effect of physical activity in pregnancy on the lipid profiles of mother and newborn, including HDL proteome. Geburtshilfe Frauenheilkd. 2014;74(S 01). doi:10.1055/s-0034-1388227

22. Coll CVN, Domingues MR, Gonçalves H, Bertoldi AD. Perceived barriers to leisure-time physical activity during pregnancy: a literature 
review of quantitative and qualitative evidence. J Sci Med Sport. 2017;20(1):17-25. PubMed ID: 27372276 doi:10.1016/j.jsams.2016. 06.007

23. Chan CWH, Au Yeung E, Law BMH. Effectiveness of physical activity interventions on pregnancy-related outcomes among pregnant women: a systematic review. Int J Environ Res Public Health. 2019;16(10):1840. doi:10.3390/ijerph16101840

24. Smith R, Reid H, Matthews A, Calderwood C, Knight M, Foster C. Infographic: physical activity for pregnant women. Br J Sports Med. 2018;52(5):532-533. doi:10.1136/bjsports-2017-098037
25. Jenum AK, Mrøkrid K, Sletner L, et al. Impact of ethnicity on gestational diabetes identified with the WHO and the modified International Association of Diabetes and Pregnancy Study Groups criteria: a population-based cohort study. Eur J Endocrinol. 2012;166(2):317324. PubMed ID: 22108914 doi:10.1530/EJE-11-0866

26. Ghiasi A, Ziaei S, Faghihzadeh S. The relationship between levels of lipids and lipoprotein B-100 in maternal serum and umbilical cord serum and assessing their effects on newborn infants anthropometric indices. J Midwifery Reprod Health. 2014;2(4):227-232. doi:10. 22038/JMRH.2014.3239 